\title{
Short Report: Common Genotypic Polymorphisms in Glutathione S-Transferases in Mild and Severe Falciparum Malaria in Tanzanian Children
}

\author{
Reginald A. Kavishe, Teun Bousema, Seif A. Shekalaghe, Robert W. Sauerwein, Frank W. Mosha, \\ Andre J. A. M. van der Ven, Frans G. M. Russel, and Jan B. Koenderink* \\ Departments of Pharmacology and Toxicology, Medical Microbiology, Internal Medicine, Radboud University Nijmegen \\ Medical Centre, Nijmegen, The Netherlands; Kilimanjaro Christian Medical College of Tumaini University, Tanzania
}

\begin{abstract}
Malaria infection induces oxidative stress in the host cells. Antioxidant enzymes such as glutathione S-transferases (GSTs) are responsible for fighting reactive oxygen species and reduction of oxidative stress. Common GST polymorphisms have been associated with susceptibility to different diseases whose pathologies involve oxidative stress. In this study, we tested the hypothesis that GST polymorphisms that lead to reduced or lack of enzyme activity are associated with severe Plasmodium falciparum malarial anemia. We studied the genotypic distribution of GSTM1, GSTT1, and GSTP1 polymorphisms between mild malaria $(N=107)$ and severe malarial anemia $(N=50)$ in Tanzanian children. We did not find a significant relationship with the GSTT1 polymorphism. GSTM1-null was higher in the severe malaria anemia group but the difference was not significant $(P=0.08)$. However, a significant association of GSTP1 I105V genotype with severe malarial anemia was discovered $(26.0 \%$ against $10.3 \%$ mild malaria, $P=0.004)$. We concluded that GSTP1 and possibly GSTM1 may protect against severe falciparum malaria in children.
\end{abstract}

Oxidative stress plays an important role in malaria immunity and pathogenesis. Malaria-induced oxidative stress is thought to originate from immuno-defensive reactions of the host cells against the parasite and as a result of parasite metabolism. The parasite feeds on hemoglobin and releases the highly reactive and toxic heme. This can react with molecular oxygen to form hemin and superoxide radical $\left(\mathrm{O}_{2}^{-}\right)$, a highly reactive oxygen species. In the parasite's food vacuole heme is, however, rendered inert and nontoxic through conversion into hemozoin, the malaria pigment. ${ }^{1}$ Most of the quinoline antimalarials interfere with the conversion of heme to hemozoin thereby inducing its accumulation inside the food vacuole and eventually killing the parasite., ${ }^{2,3}$

In severe malaria, parasite toxins may trigger the release of oxygen free radicals and stimulate a variety of proinflammatory cytokines, such as tumor necrosis factor-alpha, interleukins, gamma interferon, and nitric oxide. ${ }^{4}$ These proinflammatory factors are believed to cause much of the clinical complications observed in severe malaria with multiple organ involvement. Several studies have implicated malariainduced oxidative stress in complications such as reduced macrophage function, reduced erythrocyte deformability, and increased activation of pro-inflammatory cytokines. ${ }^{5-7}$ In children with malaria, both blood plasma and erythrocytic lipid peroxidation are increased, whereas erythrocytic antioxidants such as glutathione (GSH) were shown to be lower in patients than in controls. ${ }^{8}$ Polymorphisms resulting into absence or reduced enzyme activity have been identified and linked with pathogenesis in a number of disorders and diseases characterized with increased oxidative stress. ${ }^{9-11}$ In a previous study, we observed that GSTM1-null genotype was associated with severe malaria in Cameroonian children. ${ }^{12}$ In this study, we have investigated the genotypic distribution of human GSTM1, GSTT1, and GSTP1 polymorphisms in mild versus severe malaria in Tanzanian children.

The clinical data and DNA samples of this study were collected in the period between July and September 2006, in a drug

*Address correspondence to Jan B. Koenderink, Department of Pharmacology and Toxicology 149, Radboud University Nijmegen Medical Centre, PO Box 9101, 6500 HB Nijmegen, The Netherlands. E-mail: J.Koenderink@ncmls.ru.nl efficacy study of mild malaria cases ${ }^{13}$ in Mnyuzi, Tanga Region, Tanzania. Briefly, children 3-15 years of age with a temperature $>37.5^{\circ} \mathrm{C}$ or a history of fever within the last 48 hours and with Plasmodium falciparum mono-infection at a density between 500 and 100,000 parasites $/ \mu \mathrm{L}$ were eligible for recruitment. Children with a hemoglobin $(\mathrm{Hb})$ concentration below $8 \mathrm{~g} / \mathrm{dL}$, as measured by HemoCue (HemoCue AB, Ängelholm, Sweden), were excluded. Children with severe malaria were included for the current study in the same study period. Severe anemia ( $\mathrm{Hb}$ $<5 \mathrm{~g} / \mathrm{dL}$ ) was observed in all children who attended the clinic with severe malaria. Additional signs of severe disease that were examined: hyper parasitaemia ( $\geq 250,000$ parasites $/ \mu \mathrm{L})$, metabolic acidosis manifested by respiratory distress as described by Marsh and others, ${ }^{14}$ cerebral malaria presented as coma score $\leq 2$ (Blantyre coma scale $)^{15}$ or impaired consciousness with Blantyre score $<3$ and prostration or extreme weakness (e.g., inability to sit or stand). For severe cases treatment was initiated with quinine, according to Tanzanian National Guidelines and referred to the nearby district hospital in Korogwe in case the study physician considered this appropriate. There was no active follow-up of the outcome of severe malaria cases after the appropriate treatment was installed.

For all mild and severe malaria cases, a malaria blood slide, Hb measurement, and filter paper DNA sample were collected. A short questionnaire was used to obtain information on sex, age, disease presentation, and ethnicity of the patient. The ethical clearance for the collection of the mild malaria material was obtained from the Tanzanian National Institute for Medical Research (NIMR/HQ/R.8a Vol. XIII/446) and clearance for the collection of material of severe malaria cases was obtained from Kilimanjaro Christian Medical Center (KCMC 2006\#28). In the informed consent obtained from the parents or guardians of the children, they approved the use of their children's DNA samples to study the relation between human genetic factors and malaria disease presentation. A total of 107 mild and 50 severe malaria cases were enrolled. Parasite density in the severe malaria group ranged from 4,640-174,000 parasites $/ \mu \mathrm{L}$ and was higher than in the mild malaria group (Wilcoxon-rank sum test, $P<0.001)$. For more group characteristics see Table 1.

The DNA extracted from the dried filter papers was done using Nucleospin Tissue kits (Macherey-Nagel, Düren, 
TABLE 1

Characteristics of mild vs. severe malaria in falciparum malaria in Tanzanian children

\begin{tabular}{|c|c|c|}
\hline & Mild & Severe malaria \\
\hline$N$ & 107 & 50 \\
\hline Age, median (IQR) & $5.0(3.0-9.0)$ & $8.0(5.0-11.0)$ \\
\hline Gender, $\%$ male $(\mathrm{n} / N)$ & $51.4(55 / 107)$ & $60.0(30 / 50)$ \\
\hline $\begin{array}{l}\text { Hemoglobin concentration, } \\
\text { median g/dL (IQR) }\end{array}$ & $10.6(9.7-11.9)$ & $4.3(3.8-4.8)$ \\
\hline $\begin{array}{l}\text { Temperature, } \\
\text { median (IQR) }\end{array}$ & $37.3(36.9-38.0)$ & $38.5(37.8-39.1)$ \\
\hline $\begin{array}{l}\text { Asexual parasite } \\
\text { density, GM (IQR) }\end{array}$ & $\begin{array}{c}7,700 \\
(1,120-23,480)\end{array}$ & $\begin{array}{c}105,900 \\
(57,480-121,040)\end{array}$ \\
\hline $\begin{array}{l}\text { Signs of severe disease: } \\
\text { Severe anemia, \% }(\mathrm{n} / N) \\
\text { Hyperparasitemia, \% }(\mathrm{n} / N) \\
\text { Respiratory distress, \% }(\mathrm{n} / N) \\
\text { Reduced consciousness, \% }(\mathrm{n} / N) \\
\text { Prostration, \% }(\mathrm{n} / N)\end{array}$ & $\begin{array}{l}- \\
- \\
- \\
- \\
-\end{array}$ & $\begin{array}{c}100.0(50 / 50) \\
0.0(0 / 50) \\
6.0(3 / 50) \\
4.0(2 / 50) \\
8.0(4 / 50)\end{array}$ \\
\hline
\end{tabular}

$\mathrm{IQR}=$ interquartile range; $\mathrm{GM}=$ group median.

Germany), polymerase chain reaction (PCR) was performed using native, Taq polymerase (invitrogen) and all primers were purchased from Biolegio, Nijmegen, The Netherlands. Primers for GSTT1, GSTM1, and GSTP1 were designed according to Pemble and others, ${ }^{16}$ Bröckmoller and others, ${ }^{17}$ and Watson and others, ${ }^{18}$ respectively. The PCR conditions for GSTM1, GSTT1, and GSTP1 were followed as previously described. ${ }^{12}$ Samples that gave negative results for GSTM1 and T1 were measured again with $\beta$-globin as a control.

The results of the polymorphism analysis in mild and severe malaria are depicted in Table 2. For GSTM1 there was a higher prevalence of GSTM1-null genotype in the severe group (40\%) than in the mild group $(26.2 \%)$, although not statistically significant $(P=0.08)$. The observed distributions in mild malaria are comparable to the reported distribution of GSTM1-null in African population (22-39\%) ${ }^{19-23}$ In Caucasian population, however, the GSTM1-null frequency is higher (around 50\%) than in the African population..$^{21,23,24}$ In our previous study with 138 children from Cameroon, we found a statistically significant difference for the GSTM1-null frequency: $32 \%$ and $58 \%$ in uncomplicated malaria and severe malaria, respectively. ${ }^{12}$

TABLE 2

Genotypic and allelic distribution of glutathione S-transferases (GSTs) gene polymorphisms among mild vs. severe falciparum malaria groups in Tanzanian children

\begin{tabular}{|c|c|c|c|}
\hline & Mild malaria & Severe malaria & $P$ value \\
\hline GSTM1-null, \% (n/N) & $26.2(28 / 107)$ & $40.0(20 / 50)$ & 0.08 \\
\hline GSTT1-null, \% (n/N) & $47.7(51 / 107)$ & $54.0(27 / 50)$ & 0.46 \\
\hline \multicolumn{4}{|l|}{ GSTP1 } \\
\hline Wild type, \% (n/N) & $39.3(42 / 107)$ & $16.0(8 / 50)$ & \\
\hline $\begin{array}{l}\text { Heterozygous mutant, } \\
\quad \%(\mathrm{n} / N)\end{array}$ & $50.5(54 / 107)$ & $58.0(29 / 50)$ & \\
\hline $\begin{array}{l}\text { Homozygous mutant, } \\
\quad \%(\mathrm{n} / N)\end{array}$ & $10.3(11 / 107)$ & $26.0(13 / 50)$ & $0.004 *$ \\
\hline $\begin{array}{l}G S T M 1 \text { and } P 1 \text { combined } \\
\text { One or both enzyme }\end{array}$ & & & \\
\hline wild type, \% (n/N) & $83.2(89 / 107)$ & $64.0(32 / 50)$ & \\
\hline $\begin{array}{l}\text { Both enzymes mutant } \\
\text { (P1-hetero/homozygous), } \\
\text { \% (n/N) }\end{array}$ & $16.8(18 / 107)$ & $36.0(18 / 50)$ & 0.007 \\
\hline
\end{tabular}

The prevalence of homozygous GSTP1 I105V in the severe malaria group $(26.0 \%)$ was significantly higher than in the mild group $(10.3 \%)$. This indicates for the first time an association of the GSTP1 I105V genotype with severe malaria. There is evidence that the GSTP1 I105V polymorphism may have a substrate-dependent effect on the enzyme activity. ${ }^{18,25,26}$ The general distribution of the homozygous GSTP1 I105V genotype in the mild malaria group is comparable to previous studies in a Brazilian population of African descent $(8.3 \%)$ and Caucasians $(11.3 \%) .{ }^{21,24}$ In our previous study with Cameroonian children, ${ }^{12}$ we found in uncomplicated malaria $(21 \%)$ and severe malaria (26\%) frequencies that are comparable to what we now observe in severe malaria (26.0\%).

When a combined analysis of GSTM1 and GSTP1 was performed, the presence of wild-type condition on one or both of the two genes was $64.0 \%$ and $83.2 \%$ for the severe and mild malaria groups, respectively, and for the presence of mutations on both enzymes (hetero- or homozygous mutant) was $36.0 \%$ and $16.8 \%$ for the severe and mild malaria groups, respectively $(P=0.007)$.

The prevalence of GSTT1-null was comparable in both groups (48\% and 54\%). In the Cameroonian study we also observed no differences; although the frequencies were lower (21\% and $29 \%$ ).

This study has shown association of GSTP1 I105V, and a trend but not significant association of GSTM1, with severe malaria anemia. GSTP1 and GSTM1 are expressed in all blood cells with higher expression in lymphoid than erythroid cell types, whereas GSTT1 and GSTA are expressed in higher levels in erythrocytes than in lymphoid cells. ${ }^{27}$ In this study, we did not investigate on GSTA and we did not detect an association of GSTT1 with malaria, which is consistent with our previous observation. ${ }^{12}$ It is not clear how GST polymorphisms can affect the malaria infection outcome. The GSH is important for parasite growth and in vitro studies have documented detoxification of heme via a GSH-utilizing pathway, that can be inhibited by chloroquine and amodiaquine. ${ }^{28,29}$ Furthermore, drugs known to reduce cellular GSH were shown to potentiate the action of chloroquine in drug-resistant rodent malaria. ${ }^{30}$

Glutathione S-transferase (GST) polymorphisms can change the enzyme activity, which can lead to reduced detoxification of the host cell or increased availability of host GSH that might be used by the parasite. In both cases the malaria pathology could be accelerated. It is also likely that the impact of GSTs is not direct on erythrocytes but on other cells that are involved in the immune response mechanisms and that severe malarial anemia as an outcome can partly be attributed to such responses. Therefore, further studies including in vitro cellular studies to assess malarial outcomes for specific GST polymorphism genotypes are important.

In conclusion, GSTP1 and possibly GSTM1 may have protective effects against severe falciparum malaria in children. The contribution of specific GST polymorphisms to severe disease may differ between populations or geographic areas. These findings do not undermine the importance of oxidative stress in malaria clearance, but rather provide a broader perspective on the impact of oxidative stress on both the host and parasite cells.

Received November 27,2008. Accepted for publication April 18, 2009.

Financial support: Reginald A. Kavishe is supported by NWOWOTRO (WIZ93-465) through PRIOR. 
Authors' addresses: Reginald A. Kavishe, Frans G. M. Russel, and Jan B. Koenderink, Department of Pharmacology and Toxicology 149, Radboud University Nijmegen Medical Centre, PO Box 9101, 6500 HB Nijmegen, The Netherlands. Teun Bousema and Robert W. Sauerwein, Department of Medical Microbiology 268, Radboud University Nijmegen Medical Centre, PO Box 9101, 6500 HB Nijmegen, The Netherlands. Seif A. Shekalaghe and Frank W. Mosha, Kilimanjaro Christian Medical College of Tumaini University, PO Box 2240, Moshi, Tanzania. Andre J. A. M. van der Ven, Department of Internal Medicine 463, Radboud University Nijmegen Medical Centre, PO Box 9101, 6500 HB Nijmegen, The Netherlands.

\section{REFERENCES}

1. Pagola S, Stephens PW, Bohle DS, Kosar AD, Madsen SK, 2000. The structure of malaria pigment beta-haematin. Nature 404: 307-310.

2. Slater AF, 1993. Chloroquine: mechanism of drug action and resistance in Plasmodium falciparum. Pharmacol Ther 57: 203-235.

3. Pandey AV, Bisht H, Babbarwal VK, Srivastava J, Pandey KC, Chauhan VS, 2001. Mechanism of malarial haem detoxification inhibition by chloroquine. Biochem J 355: 333-338.

4. Maitland K, Marsh K, 2004. Pathophysiology of severe malaria in children. Acta Trop 90: 131-140.

5. Schwarzer E, Turrini F, Giribaldi G, Cappadoro M, Arese P, 1993. Phagocytosis of $P$. falciparum malarial pigment hemozoin by human monocytes inactivates monocyte protein kinase $\mathrm{C}$. Biochim Biophys Acta 1181: 51-54.

6. Schwarzer E, Arese P, 1996. Phagocytosis of malarial pigment hemozoin inhibits NADPH-oxidase activity in human monocytederived macrophages. Biochim Biophys Acta 1316: 169-175.

7. Taramelli D, Basilico N, Pagani E, Grande R, Monti D, Ghione M, Olliaro P, 1995. The heme moiety of malaria pigment (betahematin) mediates the inhibition of nitric oxide and tumor necrosis factor-alpha production by lipopolysaccharide-stimulated macrophages. Exp Parasitol 81: 501-511.

8. Becker K, Tilley L, Vennerstrom JL, Roberts D, Rogerson S, Ginsburg H, 2004. Oxidative stress in malaria parasite-infected erythrocytes: host-parasite interactions. Int $J$ Parasitol 34: 163-189.

9. Ali-Osman F, Akande O, Antoun G, Mao JX, Buolamwini J, 1997. Molecular cloning, characterization, and expression in Escherichia coli of full-length cDNAs of three human glutathione S-transferase Pi gene variants. Evidence for differential catalytic activity of the encoded proteins. J Biol Chem 272: 10004-10012.

10. Strange RC, Jones PW, Fryer AA, 2000. Glutathione S-transferase: genetics and role in toxicology. Toxicol Lett 112-113: 357-363.

11. Strange RC, Lear JT, Fryer AA, 1998. Glutathione S-transferase polymorphisms: influence on susceptibility to cancer. Chem Biol Interact 111-112: 351-364.

12. Kavishe RA, Koenderink JB, McCall MB, Peters WH, Mulder B, Hermsen CC, Sauerwine RW, Russel FG, Van der Ven AJ, 2006. Short report: severe Plasmodium falciparum malaria in Cameroon: associated with the glutathione S-transferase M1 null genotype. Am J Trop Med Hyg 75: 827-829.

13. Shekalaghe S, Drakeley C, Gosling R, Ndaro A, van Meegeren M, Enevold A, Alifrangis M, Mosha F, Sauerwine R, Bousema T, 2007. Primaquine clears submicroscopic Plasmodium falciparum gametocytes that persist after treatment with sulphadoxine-pyrimethamine and artesunate. PLoS Clin Trials 2: e1023.

14. Marsh K, Forster D, Waruiru C, Mwangi I, Winstanley M, Marsh V, Newton C, Winstanley P, Peshu N, et al., 1995. Indicators of lifethreatening malaria in African children. $N$ Engl J Med 332: 1399-1404.

15. Molyneux ME, Taylor TE, Wirima JJ, Borgstein A, 1989. Clinical features and prognostic indicators in pediatric cerebral malaria: a study of 131 comatose Malawian children. $Q J$ Med 71: 441-459.

16. Pemble S, Schroeder KR, Spencer SR, Meyer DJ, Hallier E, Bolt HM, et al., 1994. Human glutathione S-transferase theta (GSTT1): cDNA cloning and the characterization of a genetic polymorphism. Biochem J 300: 271-276.

17. Bröckmoller J, Kerb R, Drakoulis N, Nitz M, Roors I, 1993. Genotype and phenotype of glutathione S-transferase class isoenzymes and psi in lung cancer patients and controls. Lung Cancer 10: 273.

18. Watson MA, Stewart RK, Smith GBJ, Massey TE, Bell DA, 1998. Human glutathione S-transferase P1 polymorphisms: relationship to lung tissue enzyme activity and population frequency distribution. Carcinogenesis 19: 275-280.

19. Mcglynn KA, Rosvold EA, Lustbader ED, Hu Y, Clapper ML, Zhou TL, et al., 1995. Susceptibility to hepatocellular carcinoma is associated with genetic variation in the enzymatic detoxification of aflatoxin B-1. Proc Natl Acad Sci USA 92: 2384-2387.

20. Mukanganyama S, Masimirembwa CM, Naik YS, Hasler JA, 1997. Phenotyping of the glutathione S-transferase M1 polymorphism in Zimbabweans and the effects of chloroquine on blood glutathione S-transferases M1 and A. Clin Chim Acta 265: 145-155.

21. Rossini A, Rapozo DC, Amorim LM, Macedo JM, Medina R, Neto JF, Gallo CV, Pinto LF, 2002. Frequencies of GSTM1, GSTT1, and GSTP1 polymorphisms in a Brazilian population. Genet Mol Res 1: 233-240.

22. Tiemersma EW, Omer RE, Bunschoten A, van't Veer P, Kok FJ, Idris MO, Kadaru AM, Fedail SS, Kampman E, 2001. Role of genetic polymorphism of glutathione-S-transferase T1 and microsomal epoxide hydrolase in aflatoxin-associated hepatocellular carcinoma. Cancer Epidemiol Biomarkers Prev 10: 785-791.

23. Zhao L, Alldersea J, Fryer A, Tighe A, Ollier B, Thomson W, Jones P, Strange R, 1994. Polymorphism at the glutathione S-transferase GSTM1 locus: a study of the frequencies of the GSTM1-A, B, A/B and null phenotypes in Nigerians. Clin Chim Acta 225: 85-88.

24. Schneider J, Bernges U, Philipp M, Woitowitz HJ, 2004. GSTM1, GSTT1, and GSTP1 polymorphism and lung cancer risk in relation to tobacco smoking. Cancer Lett 208: 65-74.

25. Hu X, Xia H, Srivastava SK, Herzog C, Awasthi YC, Ji XH, Zimniak P, Singh SV, 1997. Activity of four allelic forms of glutathione S-transferase hGSTP1-1 for diol epoxides of polycyclic aromatic hydrocarbons. Biochem Biophys Res Commun 238: 397-402.

26. Sundberg K, Johansson AS, Stenberg G, Widersten M, Seidel A, Mannervik B, Jernström B, 1998. Differences in the catalytic efficiencies of allelic variants of glutathione transferase P1-1 towards carcinogenic diol epoxides of polycyclic aromatic hydrocarbons. Carcinogenesis 19: 433-436.

27. Wang LH, Groves MJ, Hepburn MD, Bowen DT, 2000. Glutathione S-transferase enzyme expression in hematopoietic cell lines implies a differential protective role for $\mathrm{T} 1$ and $\mathrm{A} 1$ isoenzymes in erythroid and for M1 in lymphoid lineages. Haematologica 85: 573-579.

28. Famin O, Krugliak M, Ginsburg H, 1999. Kinetics of inhibition of glutathione-mediated degradation of ferriprotoporphyrin IX by antimalarial drugs. Biochem Pharmacol 58: 59-68.

29. Ginsburg H, Famin O, Zhang JM, Krugliak M, 1998. Inhibition of glutathione-dependent degradation of heme by chloroquine and amodiaquine as a possible basis for their antimalarial mode of action. Biochem Pharmacol 56: 1305-1313.

30. Deharo E, Barkan D, Krugliak M, Golenser J, Ginsburg H, 2003. Potentiation of the antimalarial action of chloroquine in rodent malaria by drugs known to reduce cellular glutathione levels. Biochem Pharmacol 66: 809-817. 